\title{
The effect of rotation on the onset of electrohydrodynamic instability of an elastico-viscous dielectric fluid layer
}

\author{
G.C. RANA ${ }^{1 *}$, R. CHAND ${ }^{2}$, and V. SHARMA ${ }^{3}$ \\ ${ }^{1}$ Department of Mathematics, Sidharth Govt. College, Nadaun-177 005, Himachal Pradesh, India \\ ${ }^{1}$ Visiting Faculty, Department of Mathematics, Apex Professional University, Pasighat, Arunachal Pradesh, India \\ 2 Department of Mathematics, Government College Nurpur, Himachal Pradesh, India \\ ${ }^{3}$ Department of Mathematics and Statistics, Himachal Pradesh University, Shimla, India
}

\begin{abstract}
In this paper the combined effect of uniform rotation and AC electric field on the onset of instability in a horizontal layer of an elastico-viscous fluid stimulated by the dielectrophoretic force due to the variation of dielectric constant with temperature is studied. Walters' (model B') fluid model is used to describe rheological behaviour of an elastico-viscous fluid. The onset criterions for stationary and oscillatory convection are derived for the case of free-free boundaries. It is observed that Walters' (model B') fluid behaves like an ordinary Newtonian fluid and rotation has stabilizing influence whereas AC electric field has destabilizing influence on the stability of the system. The necessary condition for the occurrence of oscillatory convection is also obtained. The present results are in good agreement with the earlier published results.
\end{abstract}

Key words: Walters' (model B') fluid, rotation, AC electric field, electrohydrodynamic, viscosity, viscoelasticity.

\section{Introduction}

A comprehensive account of thermal instability of Newtonian fluid under the various assumptions of hydrodynamics and hydromagnetics has been given by Chandrasekhar [1] whereas Landau [2] has been discussed electrodynamics of continuous media and later electrohydrodynamic convection in fluids was studied by Robert [3] and Castellanos [4]. Electrohydrodynamics can be considered as a branch of fluid mechanics which deals with the effect of electrical forces. It can also be considered as that part of electrodynamics which is necessitated with the influence of moving media on electric fields. The most interesting problems in electrohydrodynamics involve both the effect of fluid in motion and the influence of the field in motion as discussed by Melcher et al. [5] whereas a review of electrodynamically enhanced heat transfer in liquids has been studied by Jones [6].

For the last few decades, considerable interest has been shown in the study of electrohydrodynamic thermal instability in dielectric fluid because it has various applications in EHD enhanced thermal transfer, EHD pumps, EHD in microgravity, micromechanic systems, drug delivery, micro-cooling system, nanotechnology etc. Chen et al. [7] discussed advances and applications of electrohydrodynamics in brief. They say that EHD heat transfer came out as an alternative method to enhance heat transfer, which is known as electrothermohydrodynamics (ETHD) in which combined action of electric field and thermal gradient for enhancing the heat transfer is considered. EHD pumps have great advantage that there is no need for a moving component such as pistons. Also it is fabricated and assembled. This type of pumps is widely used in micromechanic systems, drug delivery and micro-cooling system. Many researches have been studied the effect of AC or DC electric field on natural convection in a horizontal dielectric fluid layer by taking different types of fluids. The onset of electrohydodynamic convection in a horizontal layer of dielectric fluid was studied by Gross and Porter [8], Turnbull [9], Maekawa et al. [10], Smorodin and Velarde [11], Galal [12], Rudraiah and Gayathri [13] and Chang et al. [14].

Takashima [15] discussed the effect of uniform rotation on the onset of convective instability in a dielectric fluid under the simultaneous action of AC electric field and a vertical temperature gradient and found that the Coriolis force has an inhibiting effect on the onset of instability even when the electrical effects are taken into account and as the speed of rotation increases the coupling between the two agencies causing instability (electrical and buoyancy force) becomes tighter. Takashima and Ghosh [16] studied the electrohydrodynamic instability in a viscoelastic liquid layer and found that oscillatory modes of instability exist only when the thickness of the liquid layer is smaller than about $0.5 \mathrm{~mm}$ and for such a thin layer the force of electrical origin is much more important than buoyancy force while Takashima and Hamabata [17] studied the stability of natural convection in a vertical layer of dielectric fluid in the presence of a horizontal AC electric field. Othman [18] studied the electrohydrodynamic instability of a rotating layer of a viscoelastic fluid heated from below while the electroconvective instability in a heat generating dielectric fluid layer studied by Shivakumara et al. [19].

With the growing importance of non-Newtonian fluids having applications in geophysical fluid dynamics, chemical technology and petroleum industry attracted widespread in-

*e-mail: drgcrana@gmail.com 
G.C. Rana, R. Chand, and V. Sharma

terest in the study on non-Newtonian nanofluids. There are many elastico-viscous fluids that cannot be characterized by Maxwell's constitutive relations or by Oldroyd's constitutive relations. One such a class of fluids is Walters' (model B') elastico-viscous fluid having relevance to chemical technology and industry. Walters [20] reported that the mixture of polymethyl methacrylate and pyridine at $25^{\circ} \mathrm{C}$ containg $30.5 \mathrm{~g}$ of polymer per litre with density $0.98 \mathrm{~g}$ per litre behaves very nearly as the Walters' (model B') elastico-viscous fluid. Walters' (model B') elastic-viscous fluid form the basis for the manufacture of many important polymers and useful products. In the case of Walters' (model B') fluid, the term $\left[\mu \nabla^{2} \mathbf{q}\right]$ in the equations of motion (Chandrasekhra [1]) is replaced by the term $\left[\left(\mu-\mu^{\prime} \frac{\partial}{\partial t}\right) \nabla^{2} \mathbf{q}\right]$, where $\mu$ and $\mu^{\prime}$ are the viscosity and viscoelasticity of the incompressible Walters' (model B') fluid, $\nabla^{2}$ is the Laplacian operator and $\mathbf{q}$ is the Darcian (filter) velocity of the fluid. Rotation plays an important role as rotating system occurs in various astrophysical, geophysical and industrial processes. A good account of thermal instability problems of a rotating viscoelastic fluid is given by Sharma and Rana [21] and Shivakumara et al. [22], Rana and Jamwal [23], Chand and Rana [24] and Rana et al. [25]. Ruo et al. [26] studied the effect of rotation on the electrohydrodynamic instability of a fluid layer with an electrical conductivity gradient whereas Shivakumara et al. [27] studied the electrohydrodynamic instability of rotation couple stress dielectric fluid layer and found that the effect of increasing AC electric field number is to increase the transfer of heat more effectively and hasten the onset of convection and rotation inhibits the onset of electrohydrodynamic instability.

The growing number of applications of electrohydrodynamics and rotation, our main aim in the present paper is to study the effect of uniform rotation on electrohydrodynamic thermal instability in a Walters' (model B') fluid. To the best of my knowledge, this problem has not been studied yet.

\section{Mathematical model}

Here we consider an infinite horizontal layer of an incompressible Walters' (model B') elastico-viscous fluid of thickness $d$, bounded by the planes $z=0$ and $z=d$ as shown in Fig. 1. The layer is rotating about the vertical axis with con-

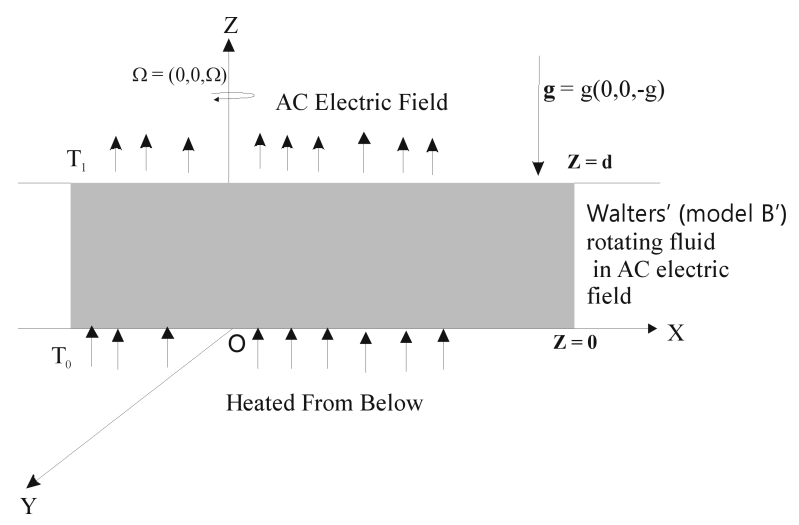

Fig. 1. Physical configuration stant angular velocity $\boldsymbol{\Omega}=(0,0, \Omega)$ and uniform vertical AC electric field applied across the layer, which is acted upon by a gravity force $\boldsymbol{g}=(0,0,-g)$ aligned in the $z$ direction. The temperature $T$ at the lower and upper boundaries is assumed to take constant values $T$ and $T_{1}(<T)$ respectively.

2.1. Governing equations. Let $\rho, \mu, \mu^{\prime}, p, K, \mathbf{q}(u, v, w)$, g, $\Omega, T, \kappa$ and $E$ denote respectively, the density, viscosity, viscoelasticity, pressure, dielectric constant, Darcy velocity vector, acceleration due to gravity, angular velocity, temperature, thermal diffusivity and the root-mean-square value of electric field. Then the equations of conservation of mass, momentum and thermal energy for Walters' (model B') elastico-viscous fluid (Chandrasekhar [1], Walters' [20], Takashima [15], Sharma and Rana [21], Rana et al. [25], and Shivakumara [27]) are

$$
\begin{gathered}
\nabla \cdot \mathbf{q}=0 \\
\rho \frac{d \mathbf{q}}{d t}=-\nabla P+\rho \mathbf{g}+\left(\mu-\mu^{\prime} \frac{\partial}{\partial t}\right) \nabla^{2} \mathbf{q} \\
+2 \rho(\mathbf{q} \times \mathbf{\Omega})-\frac{1}{2}(\mathbf{E} \cdot \mathbf{E}) \nabla K, \\
\frac{\partial T}{\partial t}+(\mathbf{q} \cdot \nabla) T=\kappa \nabla^{2} \mathrm{~T},
\end{gathered}
$$

where

$$
\frac{d}{d t}=\frac{\partial}{\partial t}+\frac{1}{\varepsilon}(\mathbf{q} \cdot \nabla)
$$

stands for convection derivative and

$$
\mathbf{P}=p-\frac{\rho}{2} \frac{\partial K}{\partial \rho}(\mathbf{E} \cdot \mathbf{E})
$$

is the modified pressure.

The Coulomb force term $\rho_{e} \mathbf{E}$, where $\rho_{e}$ is the free charge density, is of negligible order as compared with the dielectrophoretic force term for most dielectric fluids in a $60 \mathrm{~Hz} \mathrm{AC}$ electric field [Takashima (1972)]. Thus, we retain only the dielectrophoretic term, i. e. last term in Eq. (2) and neglect the Coulomb force term. Furthermore, the electrical relaxation times of most dielectric liquids appear to be sufficient long to prevent the build up of free charge at standard power line frequencies. At the same time, dielectric loss at these frequencies is very low that it makes no significant contribution to the temperature field. It is also seen that the dielectrophoretic force term depends on $(\mathbf{E} \cdot \mathbf{E})$ rather than $\mathbf{E}$. As the variation of $\mathbf{E}$ is so speedy, the root-mean-square value of $\mathbf{E}$ is used as effective value in determining the motion of fluids. So we can consider the AC electric field as the DC electric field whose strength is equal to the root mean square value of the $\mathrm{AC}$ electric field.

A charged body in an electric field tends to along the electric field lines and impart momentum to the surrounding fluid. The Maxwell equations are

$$
\begin{gathered}
\nabla \times \mathbf{E}=0, \\
\nabla \cdot(K \mathbf{E})=0 .
\end{gathered}
$$

Using Eq. (5), the electric potential can be expressed as

$$
\mathbf{E}=-\nabla V
$$


The effect of rotation on the onset of electrohydrodynamic instability of an elastico-viscous dielectric fluid layer

where $V$ is the root mean square value of electric potential. The dielectric constant is assumed to be linear function of temperature and is of the form

$$
K=K_{0}\left[1-\gamma\left(T-T_{0}\right)\right],
$$

where $\gamma>0$, is the thermal coefficient of expansion of dielectric constant and is assumed to be small.

The equation of state is

$$
\rho=\rho_{0}\left[1-\alpha\left(T-T_{0}\right)\right],
$$

where $\alpha$ is coefficient of thermal expansion and the suffix zero refers to values at the reference level $z=0$.

2.2. Basic state. The basic state of the system is taken to be quiescent layer (no settling) and is given by

$$
\begin{aligned}
\mathbf{q}=\mathbf{q}_{b}(z), & P=P_{b}(z), \quad T=T_{b}(z), \\
\mathbf{E}=\mathbf{E}_{b}(z), & K=K_{b}(z), \quad \rho=\rho_{b}(z),
\end{aligned}
$$

where the subscript $b$ denotes the basic state.

Substituting equations given in (10) in Eqs. (1)-(9), we obtain

$$
\begin{gathered}
0=-\nabla \frac{P_{b}(z)}{\rho_{0}}+\frac{\rho_{b}(z)}{\rho_{0}} \mathbf{g}-\frac{1}{2 \rho_{0}}\left(\mathbf{E}^{2}\right) \nabla K, \\
\frac{d^{2} T_{b}(z)}{d z^{2}}=0, \\
K_{b}(z)=K_{0}\left[1-\gamma\left(T_{b}-T_{0}\right)\right], \\
\rho_{b}(z)=\rho_{0}\left[1-\alpha\left(T_{b}-T_{0}\right)\right], \\
\nabla \cdot\left(K_{b} E_{b}\right)=0 .
\end{gathered}
$$

Solving Eq. (12) by using the following boundary conditions

$$
\begin{gathered}
\quad T_{b}(z)=T_{0} \quad \text { at } \quad z=0 \\
\text { and } \quad T_{b}(z)=T_{1} \quad \text { at } \quad z=1,
\end{gathered}
$$

we obtain

$$
T_{b}=T_{0}-\Delta T z / d .
$$

In view of Eq. (15) and noting that $E_{b x}=E_{b y}=0$. It follows that

$$
K_{b} E_{b z}=K_{0} E_{0}=\text { constant (say). }
$$

Then

$$
\mathbf{E}=\mathbf{E}_{b}(z)=\frac{E_{0}}{1+\gamma \Delta T z / d}
$$

Hence

$$
V_{b}(z)=-\frac{E_{0} d}{\gamma \Delta T} \log (1+\gamma \Delta T z / d),
$$

where

$$
E_{0}=-\frac{V_{1} \gamma \Delta T / d}{\log (1+\gamma \Delta T)}
$$

is the root-mean-square value of the electric field at $z=0$.

These results are identical with the results obtained by Shivakumara et al. [27].
2.3. Perturbation solutions. To study the stability of the system, we superimposed infinitesimal perturbations on the basic state, so that

$$
\begin{gathered}
\mathbf{q}=\mathbf{q}^{\prime}, \quad T=T_{b}+T^{\prime}, \\
\mathbf{E}=\mathbf{E}_{b}+\mathbf{E}^{\prime}, \quad \rho=\rho_{b}+\rho^{\prime}, \\
K=K_{b}+K^{\prime}, \quad P=P_{b}+P^{\prime},
\end{gathered}
$$

where $\mathbf{q}^{\prime}, T^{\prime}, \mathbf{E}^{\prime}, \rho^{\prime}, K^{\prime}, P^{\prime}$ be the perturbations in $\mathbf{q}, T$, $\mathbf{E}^{\prime}, \rho, K^{\prime}, P^{\prime}$ respectively. Substituting Eq. (10) in Eqs. (1)(9), linearizing the equations by neglecting the product of primed quantitities, eliminating the pressure from the momentum Eq. (2) by operating curl twice and retaining the vertical component and non-dimensionalizing the resulting equations by introducing the dimensionless variables as follows:

$$
\begin{gathered}
\left(x^{\prime}, y^{\prime}, z^{\prime}\right)=\left(\frac{x, y, z}{d}\right), \quad \mathbf{q}^{\prime}=\frac{d}{\kappa} \mathbf{q}, \\
t^{\prime}=\frac{\kappa}{d^{2}} t, \quad T^{\prime}=\frac{1}{\Delta T} T, \\
\xi^{\prime}=\frac{d^{2}}{\kappa} \xi, \quad V^{\prime}=\frac{1}{\gamma \mathrm{E}_{0} \Delta T d} V .
\end{gathered}
$$

Neglecting the primes for simplicity, we obtain the linear stability equations in the form

$$
\begin{gathered}
{\left[\frac{1}{\operatorname{Pr}} \frac{\partial}{\partial t}-\left(1-F \frac{\partial}{\partial t}\right) \nabla^{2}\right] \nabla^{2} w} \\
=R a_{t} \nabla_{h}^{2} T-\sqrt{T a} \frac{\partial \xi}{\partial z}+R a_{e} \nabla_{h}^{2}\left(T-\frac{\partial V}{\partial z}\right), \\
{\left[\frac{1}{\operatorname{Pr}} \frac{\partial}{\partial t}-\left(1-F \frac{\partial}{\partial t}\right) \nabla^{2}\right] \xi=\sqrt{T a} \frac{\partial w}{\partial z},} \\
{\left[\frac{\partial}{\partial t}-\nabla^{2}\right] T=w} \\
\nabla^{2} V=\frac{\partial T}{\partial z},
\end{gathered}
$$

where we have dimensionless parameters as:

$$
\operatorname{Pr}=\frac{\nu}{\kappa},
$$

$$
F=\frac{\mu^{\prime}}{\mu}
$$

$$
T a=\frac{4 \Omega^{2} d^{4}}{\nu^{2}}
$$

$$
\begin{gathered}
R a_{e}=\frac{\gamma^{2} K_{0} E_{0}^{2}(\Delta T)^{2} d^{2}}{\mu \kappa}, \\
\xi=\frac{\partial v}{\partial x}-\frac{\partial u}{\partial y} .
\end{gathered}
$$


G.C. Rana, R. Chand, and V. Sharma

The parameter $\operatorname{Pr}$ is The Prandtl number, $F$ is the viscoelasticity parameter and $T a$ is the Taylor number, while $R a_{t}$ is the familiar thermal Rayleigh number, $R a_{e}$ is the $\mathrm{AC}$ electric Rayleigh number and $\xi$ is the $z$-component of vorticity.

Here we assume that the temperature at the boundaries is kept fixed, the fluid layer is confined between two boundaries. The boundary conditions appropriate (Chandrasekhar [1], Takashima [15], Rana and Jamwal [23] and Shivakumara et al. [27]) to the problem are

$$
\begin{gathered}
w=\frac{\partial^{2} w}{\partial z^{2}}=\frac{\partial \xi}{\partial z}=\frac{\partial V}{\partial z}=0 \\
T=0 \quad \text { or } \quad D T=0
\end{gathered}
$$

\section{Linear stability analysis}

Following the normal mode analyses, we assume that the perturbation quantities have $x, y$ and $t$ dependence of the form

$$
\begin{gathered}
{[w, T, V, \xi]=[W(z), \Theta(z), \Phi(z), Z(z)]} \\
\cdot \exp (i l x+i m y+\omega t),
\end{gathered}
$$

where $l$ and $m$ are the wave numbers in the $x$ and $y$ direction, respectively, and $\omega$ is the complex growth rate of the disturbances.

Substituting Eq. (32) in Eqs. (23)-(26) and (31), we get

$$
\begin{aligned}
& {\left[\frac{\omega}{\operatorname{Pr}}-(1-F \omega)\left(D^{2}-a^{2}\right)\right]\left(D^{2}-a^{2}\right) W } \\
= & -R a_{t} a^{2} \Theta-\sqrt{T a} D Z+R a_{e} a^{2}(\Theta-D \Phi),
\end{aligned}
$$

$$
\begin{gathered}
{\left[\frac{\omega}{\operatorname{Pr}}-(1-F \omega)\left(D^{2}-a^{2}\right)\right] Z=\sqrt{T a} D W,} \\
{\left[\omega-\left(D^{2}-a^{2}\right)\right] \Theta=W,} \\
\left(D^{2}-a^{2}\right) \Phi=D \Theta, \\
w=D^{2} W=D Z=D \Phi=0, \\
\Theta=0 \quad \text { or } \quad D \Theta=0,
\end{gathered}
$$

where

$$
a^{2}=l^{2}+m^{2}, \quad D=\frac{d}{d z} .
$$

Equations (33)-(36) form a double eigenvalue problem for $R a_{t}$ or $R a_{e}$ and $\omega$ with respect to the boundary conditions (37).

We assume the solution to $W, \Theta, \Phi$ and $Z$ of the form

$$
\begin{aligned}
& W=W_{0} \sin \pi z, \\
& \Theta=\Theta_{0} \sin \pi z, \\
& \Phi=\Phi_{0} \cos \pi z, \\
& Z=Z_{0} \cos \pi z,
\end{aligned}
$$

which satisfy the boundary conditions of Eq. (37). Substituting Eq. (38) into Eqs. (33)-(36), we obtain the following matrix equation

$$
\left[\begin{array}{cccc}
\left(\frac{1}{\mathrm{Pr}}-F J^{2}\right) J^{2} \omega+J^{4} & -a^{2}\left(R a_{t}+R a_{e}\right) & \pi \sqrt{T a} & -R a_{e} a^{2} \pi \\
-1 & \omega+J^{2} & 0 & 0 \\
-\pi \sqrt{T a} & 0 & \left(\frac{1}{\mathrm{Pr}}-F J^{2}\right) \omega+J^{2} & 0 \\
0 & \pi & 0 & J^{2}
\end{array}\right]\left[\begin{array}{c}
W_{0} \\
\Theta_{0} \\
Z_{0} \\
\Phi_{0}
\end{array}\right]=\left[\begin{array}{c}
0 \\
0 \\
0 \\
0
\end{array}\right],
$$

where $J^{2}=\pi^{2}+a^{2}$ is the total wave number.

The linear system (39) has a non-trivial solution if and only if

$$
\left|\begin{array}{cccc}
\left(\frac{1}{\operatorname{Pr}}-F J^{2}\right) J^{2} \omega+J^{4} & -a^{2}\left(R a_{t}+R a_{e}\right) & \pi \sqrt{T a} & -R a_{e} a^{2} \pi \\
-1 & \omega+J^{2} & 0 & 0 \\
-\pi \sqrt{T a} & 0 & \left(\frac{1}{\operatorname{Pr}}-F J^{2}\right) \omega+J^{2} & 0 \\
0 & \pi & 0 & J^{2}
\end{array}\right|=0
$$

which yields

$$
R a_{t}=\frac{J^{2}\left(J^{2}+\omega\right)}{a^{2}}\left[\left(\frac{1}{\operatorname{Pr}}-F J^{2}\right) \omega+J^{2}\right]+\frac{\pi^{2} T a}{a^{2}} \frac{J^{2}+\omega}{\left(\frac{1}{\operatorname{Pr}}-F J^{2}\right) \omega+J^{2}}-\frac{a^{2}}{J^{2}} R a_{e} .
$$


Equation (40) is the dispersion relation accounting for the effect of Prandtl number, electric Rayleigh number, Taylor number and kinematic visco-elasticity parameter in a layer of Walters' (model B') elastic-viscous dielectric fluid.

Setting $\omega=i \omega_{i}$ in Eq. (40) and clearing the complex quantities from the denominator, we obtain

$$
R a_{t}=\Delta_{1}+i \omega_{i} \Delta_{2}
$$

where

$$
\begin{gathered}
\Delta_{1}=\frac{J^{2}}{a^{2}}\left[J^{4}-\left(\frac{1}{\operatorname{Pr}}-F J^{2}\right) \omega_{i}^{2}\right] \\
+\frac{\pi^{2} T a}{a^{2}}\left[\frac{J^{4}+\left(\frac{1}{\operatorname{Pr}}-F J^{2}\right) \omega_{i}^{2}}{J^{4}+\left(\frac{1}{\operatorname{Pr}}-F J^{2}\right)^{2} \omega_{i}^{2}}\right]-\frac{a^{2}}{J^{2}} R a_{e}
\end{gathered}
$$

and

$$
\begin{array}{r}
\Delta_{2}=\frac{J^{2}}{a^{2}}\left[J^{2}+\left(\frac{1}{\mathrm{Pr}}-F J^{2}\right) J^{2}\right. \\
\left.-\pi^{2} T a \frac{J^{2}-\left(\frac{1}{\mathrm{Pr}}-F J^{2}\right)}{J^{4}+\left(\frac{1}{\operatorname{Pr}}-F J^{2}\right)^{2} \omega_{i}^{2}}\right] .
\end{array}
$$

Since $R a_{t}$ is a physical quantity, it must be a real value. Hence, it follows from Eq. (43) that either $\omega_{i}=0$ (exchange stability, steady onset) or $\Delta_{2}=0 \quad \omega_{i} \neq 0$ (overstability, oscillatory onset).

\section{Stationary convection}

For stationary convection, putting $\omega=0$ in Eq. (40) reduces it to

$$
R a_{t}=\frac{\left(\pi^{2}+a^{2}\right)^{3}}{a^{2}}+\frac{\pi^{2} T a}{a^{2}}-\frac{a^{2}}{\pi^{2}+a^{2}} R a_{e} .
$$

Equation (44) expresses the thermal Rayleigh number as a function of the dimensionless resultant wave number $a$ and the parameters $T a$ and $R a_{e}$. It is found that the kinematic viscoelasticity parameter $\mathrm{F}$ vanishes with $\omega$ and the Walters' (model B') elastico-viscous dielectric fluid behaves like an ordinary Newtonian dielectric fluid. Equation (44) is identical to that obtained by Shivakumara et al. [27] in the absence couple stress parameter.

In the absence of $\mathrm{AC}$ electric field (i. e., when $R a_{e}=0$ ), Eq. (44) reduces to

$$
R a_{t}=\frac{\left(\pi^{2}+a^{2}\right)^{3}}{a^{2}}+\frac{\pi^{2} T a}{a^{2}}
$$

which is exactly the same equation as derived by Chandrasekhar [1] and Shivakumara [27].

In the absence of rotation (i.e., when $T a=0$ ), Eq. (44) reduces to

$$
R a_{t}=\frac{\left(\pi^{2}+a^{2}\right)^{3}}{a^{2}}-\frac{a^{2}}{\pi^{2}+a^{2}} R a_{e}
$$

Equation (46) is in good agreement with the equation obtained by Roberts [3] and Shivakumara [27].

To find the critical value of $R a_{t}$, we differentiate Eq. (44) with respect $a^{2}$ and equate to zero to obtain a polynomial in $a_{c}^{2}$ in the form

$$
\begin{gathered}
2\left(a_{c}^{2}\right)^{5}+5 \pi^{2}\left(a_{c}^{2}\right)^{4}+5 \pi^{4}\left(a_{c}^{2}\right)^{3} \\
+\pi^{2}\left(2 \pi^{4}-T a-R a_{e}\right)\left(a_{c}^{2}\right)^{2} \\
+\pi^{4}\left(5 \pi^{4}-2 T a\right)\left(a_{c}^{2}\right) \\
-\pi^{6}\left(\pi^{4}+T a\right)=0 .
\end{gathered}
$$

From Eq. (47), it is observed that the critical wave number varies with $T a$ and $R a_{e}$ which is identical with the equation obtained by Shivakumara [27] in the absence of couple stress parameter.

To study the effect of rotation and AC electric field on electrohydrodynamic stationary convection, we examine the behaviour of $\frac{\partial R a_{t}}{\partial T a}$ and $\frac{\partial R a_{t}}{\partial R a_{e}}$ analytically.

From Eq. (44), we obtain

$$
\frac{\partial R a_{t}}{\partial T a}=\frac{\pi^{2}}{a^{2}}
$$

which is positive, therefore, rotation inhibits the onset of electrohydrodynamic stationary convection implying thereby rotation has stabilizing effect on the system which is an agreement with the results derived by Takashima [16], Sharma and Rana [20] Rana and Jamwal [21] and Shivakumara [27].

It is evident from Eq. (44) that

$$
\frac{\partial R a_{t}}{\partial R a_{e}}=-\frac{a^{2}}{\pi^{2}+a^{2}},
$$

which is negative implying thereby $\mathrm{AC}$ electric field hastens the electroconvection implying thereby $\mathrm{AC}$ electric field has destabilizing effect on the system which is in an agreement with the results derived by Takashima [16] and Shivakumara [27].

The dispersion relation (44) is analyzed numerically. Graphs have been plotted by giving some numerical values to the parameters, to depict the stability characteristics.

In Fig. 2, the thermal Rayleigh number $R a_{t}$ is plotted against dimensionless wave number $a$ for fixed value of electric Rayleigh number (i.e., $R a_{e}=500$ ) and for different values Taylor number ( $T a)$ as shown. This shows that as Ta increases the thermal Rayleigh number $R a_{t}$ also increases. Thus rotation has stabilizing effect on stationary convection which is in good agreement with the result obtained analytically from Eq. (48).

In Fig. 3, the thermal Rayleigh number $R a_{t}$ is plotted against dimensionless wave number $a$ for fixed value Taylor number $(T a=1000)$ and for different values of electric Rayleigh number $\left(R a_{e}\right)$ as shown. This shows that as $\left(R a_{e}\right)$ increases the thermal Rayleigh number $R a_{t}$ decreases. Hence AC electric field has destabilizing effect on stationary convection which is in good agreement with the result obtained analytically from Eq. (49). 
G.C. Rana, R. Chand, and V. Sharma

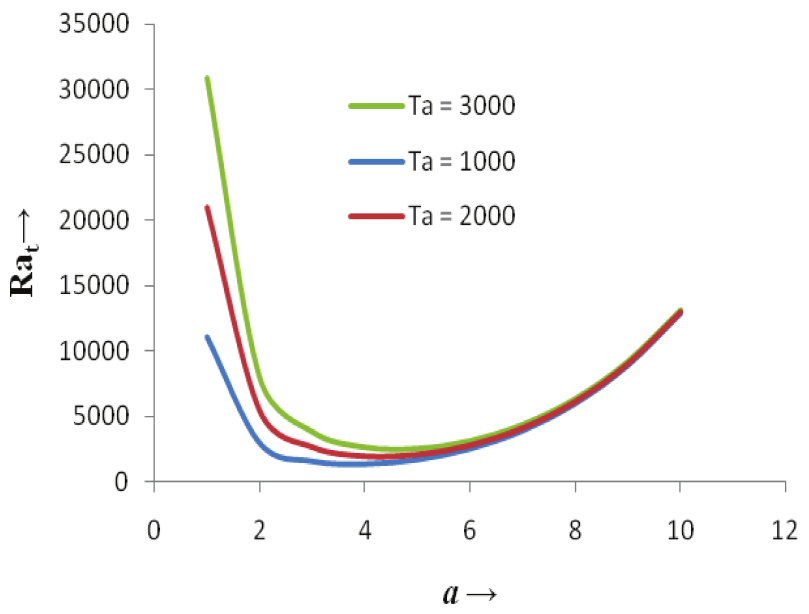

Fig. 2. Variation of thermal Rayleigh number $R a_{t}$ with the wave number $a$ for different values of the Taylor number $T a$

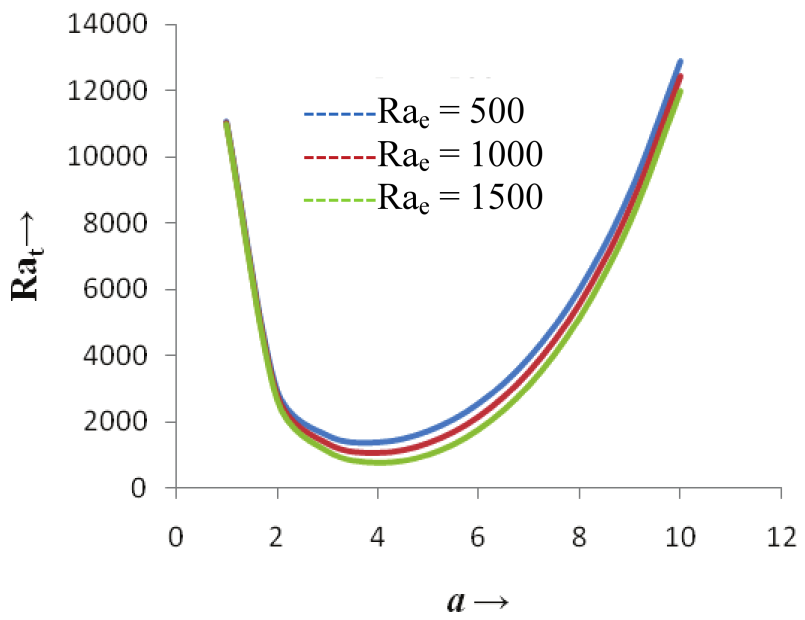

Fig. 3. Variation of thermal Rayleigh number $R a_{t}$ with the wave number $a$ for different values of AC electric Rayleigh number $R a_{e}$

\section{Oscillatory convection}

The onset of oscillatory convection corresponds to $\Delta_{2}=0$ and $\omega \neq 0$ gives an expression for the frequency of oscillations $\omega_{i}^{2}$ as

$$
\omega_{i}^{2}=\frac{\operatorname{Pr}^{2}}{\left(1-\operatorname{Pr} F J^{2}\right)^{2}}\left[-J^{4}+\frac{\pi^{2} T a}{J^{2}} \cdot \frac{\operatorname{Pr}-1+F \operatorname{Pr} J^{2}}{1-F \operatorname{Pr} J^{2}+\operatorname{Pr}}\right] .
$$

Since $\omega_{i}^{2}>0$ for the occurrence of oscillatory convection, the necessary condition for the occurrence of oscillatory convection is

$$
T a>\frac{J^{6}}{\pi^{2}}\left(\frac{\operatorname{Pr}+1-F \operatorname{Pr} J^{2}}{F \operatorname{Pr} J^{2}+\operatorname{Pr}-1}\right) .
$$

Thus, it is noted that the necessary condition depends upon the viscoelasticity parameter but independent of AC electric field. Beside the presence of viscoelasticity reduces the range of Prandtl number for oscillatory convection. The above condition is identical with the condition given by Chandrasekhar [1], when $F=0$. Eliminating $\omega_{i}^{2}$ from Eq. (41) by using Eq. (50) and noting that $\Delta_{2}=0$, we get an expression for the Rayleigh number for the onset of oscillatory convection in the form

$$
\left(R a_{t}\right)_{o s c}=\frac{2 \pi^{2} T a\left\{1+a^{*}\right\}}{a^{2} \operatorname{Pr}\left\{a^{*}\right\}\left\{1+a^{*}\right\}^{2}}-\frac{a^{2}}{\pi^{2}+a^{2}} R a_{e},
$$

where

$$
a^{*}=\frac{1}{\operatorname{Pr}}-F\left(\pi^{2}+a^{2}\right) .
$$

Equation (52) is identical with the expression given by Shivakumara et al. [27] in the absence of viscoelasticity parameter $F$. It is noted from Eq. (52) that oscillatory convection depends upon rotation, Prandtl number, viscoelasticity and $\mathrm{AC}$ electric field whereas in stationary convection Rayleigh number depends only on rotation and AC electric field.

In the absence of viscoelasticity and $\mathrm{AC}$ electric field (i. e., $F=0, R a_{e}=0$ ), Eq. (52) reduces to

$$
\left(R a_{t}\right)_{o s c}=\frac{2 \pi^{2} T a}{a^{2}\left(1+\frac{1}{\mathrm{Pr}}\right)}
$$

which is identical with the expression derived by Chandrasekhar [1].

\section{Conclusions}

The effect of rotation on the onset of electrohydrodynamic instability of Walters' (model B') elastico-viscous dielectric fluid layer heated from below has been investigated for the case of free-free boundaries by using perturbation theory and linear stability analysis based on normal modes. The main conclusions are as follow:

1. For the case of stationary convection, the non-Newtonian electrohydrodynamic Walters' (model B') elastico-viscous dielectric fluid behaves like an ordinary Newtonian fluid.

2. Rotation inhibits the onset of electrohydrodynamic stationary convection as $\partial R a_{t} / \partial T a>0$ indicating that thermal Rayleigh number $R a_{t}$ is an increasing function of Taylor number $\mathrm{Ta}$. Thus rotation has stabilizing effect on the stationary convection. Figure 2 clearly depicts the stabilizing effect of rotation.

3. AC electric field hasten the onset of electrohydrodynamic stationary convection as $\partial R a_{t} / \partial R a_{e}<0$ indicating that the thermal Rayleigh number $R a_{t}$ is an decreasing function of electric Rayleigh number $R a_{e}$. Thus AC electric field has destabilizing effect on the stationary convection. Figure 3 clearly depicts the destabilizing effect of $\mathrm{AC}$ electric field.

4. The necessary condition for the occurrence of oscillatory convection is obtained and is given by Eq. (51).

Acknowledgements. Authors would like to thank the learned referee for their valuable comments and suggestions for the improvement of quality of the paper.

\section{REFERENCES}

[1] S. Chandrasekhar, Hydrodynamic and Hydromagnetic Stability, Dover Publication, New York, 1961.

[2] L.D. Landau, Electrodynamics of Continuous Media, Oxford, 1960. 
The effect of rotation on the onset of electrohydrodynamic instability of an elastico-viscous dielectric fluid layer

[3] P.H. Roberts, "Electrohydrodynamic convection", Q.J. Mechanics and Applied Mathematics 22, 211-220 (1969).

[4] A. Castellanos, Electrohydrodynmics, Springer-Verlag, New York, 1998.

[5] J.R. Melcher and G.I. Taylor, "Electrohydrodynamics: a review of the role of interfacial shear stresses", Annu. Rev. Fluid Mech. 1, 11-146 (1969).

[6] T.B. Jones, "Electrohydrodynamically enhanced heat transfer in liquids-a review", in Advances in Heat Transfer, eds. T.F. Irvine Jr. \& J.P. Hartnett, pp. 107-144, Academic Press, London, 1978.

[7] X. Chen, J. Cheng, and X. Yin, "Advances and applications of electrohydrodynamics", Chinese Science Bulletin 48, 10551063 (2003).

[8] M.J. Gross and J.E. Porter, "Electrically induced convection in dielectric liquids", Nature 212, 1343-1345 (1966).

[9] R.J. Turnbull, "Effect of dielectrophoretic forces on the Benard instability", Phys. Fluids 12, 1809-1815 (1969).

[10] T. Maekawa, K. Abe, and I. Tanasawa, "Onset of natural convection under an electric field", Int. J. Heat Mass Trans. 35, 613-621 (1992).

[11] B.L. Smorodin, "Stability of plane flow of a liquid dielectric in a transverse alternating electric field", Fluid Dynamics 36, 548-555 (2001).

[12] M.M. Galal, "Electrohydrodynamic instability if two superposed viscous miscible streaming liquids", J. Electrostatics 40, 185-190 (1997).

[13] N. Rudraiah and M.S. Gayathri, "Effect of thermal modulation on the onset of electrothermoconvection in a dielectric fluid saturated porous medium", ASME J. Heat Tranfer 131, 101009-101015 (2009).

[14] M.H. Chang, A.C. Ruo, and F. Chen, "Electrohydrodynamic instability in a horizontal fluid layer with electrical conductivity gradient subject to a weak shear flow", J. Fluid Mech. 634, 191-215 (2009).

[15] M. Takashima, "The effect of rotation on electrohydrodynamic instability", Canadian J. Physics 54, 342-347 (1976).

[16] M. Takashima and A.K. Ghosh, "Electrohydrodynamic instability in a viscoelastic liquid layer", J. Phys. Soc. Japan 47, 1717-1722 (1979).
[17] M. Takashima and H. Hamabata, "The stability of natural convection in a vertical layer of dielectric fluid in the presence of a horizontal ac electric field", J. Phys. Soc. Japan 53, 17281736 (1984).

[18] M.I. Othman, "Electrohydrodynamic instability of a rotating layer of a viscoelastic fluid heated from below", Zeitschriftfür Angewandte Mathematik und Physik 55, 468-482 (2004).

[19] I.S. Shivakumara, M.S. Nagashree, and K. Hemalatha, "Electroconvective instability in a heat generating dielectric fluid layer", Int. Communications in Heat and Mass Transfer 34, 1041-1047 (2007).

[20] K. Walters', "The solution of flow problems in the case of materials with memory", J. Mecanique 1, 469-778 (1962).

[21] V. Sharma and G.C. Rana, "Thermal instability of a Walters' (Model B') elastico-viscous fluid in the presence of variable gravity field and rotation in porous medium", J. Non-Equilib. Thermodyn. 26, 31-40 (2001).

[22] I.S. Shivakumara, J. Lee, M.S. Malashetty, and S. Sureshkumara, "Effect of thermal modulation on the onset of thermal convection in Walters' B viscoelastic fluid in a porous medium”, Transport in Porous Media 87, 291-307 (2011).

[23] G.C. Rana and H.S. Jamwal, "Effect of rotation on the onset of compressible viscoelastic fluid saturating a darcy-brinkman porous medium", Engineering Mechanics 19, 445-455 (2012).

[24] R. Chand and G.C. Rana, "On the onset of thermal convection in rotating nanofluid layer saturating a Darcy-Brinkman porous medium", Int. J. Heat Mass Transfer 55, 5417-5424 (2012).

[25] G.C. Rana, S.K. Kango, and S. Kumar, "Effect of rotation on the onset of convection in Walters' (model B') heated from below in a Brinkman porous medium", J. Porous Media 15, 1149-1153 (2012).

[26] A.C. Ruo, M.H. Chang, and F. Chen, "Effect of rotation on the electrohydrodynamic instability of a fluid layer with an electrical conductivity gradient", Physics of Fluids 22, 024102-1024102-11 (2010).

[27] I.S. Shivakumara, M. Akkanagamma, and Chiu-on Ng, "Electrohydrodynamic instability of a rotating couple stress dielectric fluid layer", Int. J. Heat Mass Transfer 62, 761-771 (2013). 